\title{
CHARACTERISTICS OF NON-EQUILIBRIUM ARC PLASMA IN PLASMATRON NOZZLE CHANNEL
}

\author{
A.V. IGNATOV ${ }^{1,2}$, I.V. KRIVTSUN ${ }^{1}$ and I.L. SEMENOV ${ }^{3}$ \\ ${ }^{1}$ Guangdong General Research Institute of Industrial Technology \\ (Guangzhou Research Institute of Non-Ferrous Metals), Guangzhou, PRC \\ ${ }^{2}$ E.O. Paton Electric Welding Institute, NASU \\ 11 Kazimir Malevich Str., 03680, Kiev, Ukraine. E-mail: office@paton.kiev.ua \\ ${ }^{3}$ German Aerospace Center (DLR) \\ Munich, Germany. E-mail: igor_semenov@list.ru
}

\begin{abstract}
A mathematical model was developed for processes of energy, pulse, mass and discharge transfer in non-equilibrium plasma of electric arc, burning in cylindrical channel with water-cooled walls being blown out by laminar flow of plasma inert gas. The model is based on multi-fluid equations for nonisothermal, ionization non-equilibrium arc plasma, considering double ionization of atoms of the plasma gas written in a drift-diffusion approximation. Such an approach allows uniform description of the processes taking place in central region of the channel (in arc column plasma) as well as near-wall region (in plasma ionization layer) up to interface of space charge layer directly adjacent to channel wall. Consideration of the processes taking place in collisionless layer of space discharge and determination of characteristics of thermal and electric interaction of arc plasma with channel wall is carried out using corresponding boundary conditions on interface of the indicated layer. Besides, if presence of doubly charged ions in arc plasma is taken into account, it is possible to calculate its characteristics in a wide range of values of arc current and channel radius. Numerical solution of equations of the proposed model is carried out by finite volume method. Corresponding software is developed for computer realization. Detailed numerical analysis is given to radial distribution of characteristics of argon arc plasma in the cylindrical channels of direct plasmatron nozzle as well as intensity of longitudinal electric field in arc plasma and heat flow from plasma to channel wall at different values of arc current, channel radius and consumption of plasma gas. It is shown that in contrast to the central regions of the channel, where arc plasma is virtually equilibrium, a significant thermal and ionization non-equilibrium of plasma is realized in the near-wall region. It is also shown that increase of arc current and reduction of channel radius requires consideration of the doubly charge ions present in arc plasma. Comparison of results of modelling of characteristics of the non-equilibrium argon arc plasma in the plasmatron nozzle channel with available experimental data was carried out. 11 Ref., 1 Table, 9 Figures.
\end{abstract}

Ke y word s: arc plasmatron, plasma-shaping channel, electric arc in channel, non-equilibrium plasma, mathematical modelling

Development of new and improvement of existing plasma technologies, such, for example, as plasma welding, cutting, powder surfacing and coating deposition require reliable information on integral and distributed characteristics of plasma, generated by direct and indirect arc plasmatrons depending on type and structural peculiarities of the plasmatron, mode of its operation and composition of plasma gas. Thermal, gas-dynamic and electromagnetic characteristics of arc plasma flow, generated by such devices, are mainly determined by its interaction with a wall of plasmatron plasma-shaping channel. Besides, specified interaction determines the characteristics of heat and electric effect of arc plasma on the channel wall. They allow optimizing plasmatron design and increase its operation life. Experimental determination of the characteristics of arc plasma in the plasmatron channel as well as characteristics of its interaction with the channel wall is complicated due to small geometrical dimensions of the channel, high values of plasma and wall temperature.

Therefore, aim of the present work is a development of mathematical model and accurate numerical research of the processes of energy, pulse, mass and discharge transfer in non-equilibrium plasma of electric arc, burning in plasmatron cylindrical channel, as well as determination of heat and electric characteristics of its interaction with the channel wall.

The model based on multi-fluid equations for thermal and ionization non-equilibrium plasma [1] can be used for theoretical description of the processes of energy, mass and electric transfer in arc plasma volume, containing electrons, ions and neutral atoms. An approach, similar to one proposed in works [2-6] for investigation of cathode and anode processes in the electric arc, including with doubly and triply charged ions, can be used for consideration of the processes of interaction of such a plasma with the channel wall.

Corresponding approaches were earlier used for numerical analysis of distributed characteristics of 
arc plasma in nozzle channel of the direct plasmatron $[7,8]$. However, used in these works channel wall boundary conditions were model ones without consideration of real structure of plasma near-wall region. It did not allow adequate description of the processes of heat and electric interaction of arc plasma with the channel wall. It should be noted that work [8] is one of the several publications, where the results of measurements of concentration distribution and electrons temperature as well as intensity of electric field for arc plasma in channel at different current values are provided together with calculation data.

Problem statement and applied approximations. Let's consider plasma of a stationary electric arc in asymptotic region [7] of extended (segmented) cylindrical channel of radius $R$, blown out by lamellar flow of inert gas at volume velocity $G$ (under normal conditions). Arc current is denoted as $I$, gas pressure in studied region of the channel $p$ is atmospheric, temperature of water-cooled (non-evaporating) channel wall is termed $T_{w}$. Assuming that given plasma system is an axisymmetric, we are going to use a cylindrical system of coordinates $(r, z)$, axis $O Z$ of which matches with the channel axis and directed as shown in Figure 1. It is supposed under these conditions that plasma gas moves along the indicated axis and electric current has an opposite direction.

Studied arc plasma can be conventionally divided on three regions [3]. The first of them is a layer of space charge directly attached to the channel wall (see Figure 1), where condition of quasineutral plasma is violated and significant part of potential drop between arc column plasma and channel surface is formed. This layer can be considered as collisionless, since thickness of this layer $R-r_{s}$, being comparable with Debye radius $r_{\mathrm{D}} \sim 10^{-8}-10^{-7} \mathrm{~m}$, is significantly less than typical lengths of free path of plasma particle $l \sim 10^{-6}-10^{-4} \mathrm{~m}$ under atmospheric pressure and typical values of temperature of arc plasma electrons $T_{\mathrm{e}} \sim 1.5 \mathrm{eV}$ [8] (corresponding typical lengths for argon plasma of atmospheric pressure are given in Figure 2).

The second region (see Figure 1) is an ionization region of nonisothermal quasineutral plasma (prelayer), where generation of charged particles takes place due to ionizing by plasma electrons of atoms of plasma gas being desorbed from the channel wall. Ions formed here are accelerated aside to the wall by electric field created by more movable electrons, and being recombinated on its surface. Thus, conditions of local ionization equilibrium are violated in the pre-layer limits. Besides, the rest of potential drop between arc column and channel wall is formed here, which can exceed corresponding drop in the space charge layer.

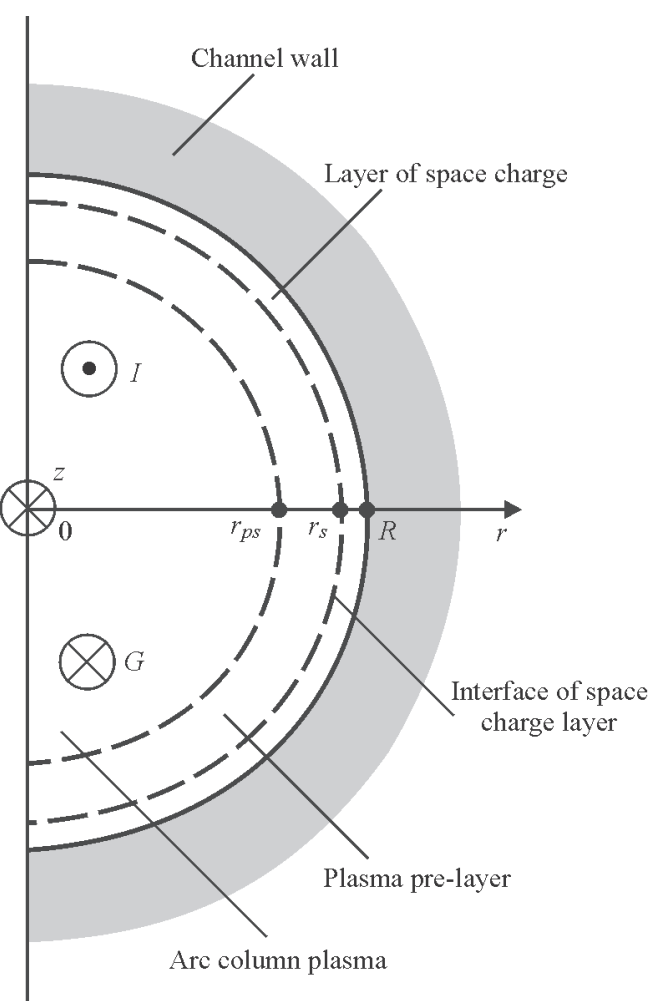

Figure 1. Scheme of arc plasma in plasmatron nozzle channel

Ionization region interface goes at $R-r_{p s}$ distance from the wall, being equal several length of free path of plasma particles. It will be matched with outer interface of plasma near-wall layer, and from it the third zone, namely arc column, will originate (see Figure 1). It is the zone, where local thermodynamic, including ionization, equilibrium takes place.

Due to the fact that present work studies arc plasma in asymptotic region of the channel, the description of processes of energy, mass and electric transfer in such a system will neglect the changes of plasma characteristics in axial direction (along the channel axis) in comparison with their radial changes. Also, taking into account the fact that studied arc is stationary, the changes of characteristics of arc plasma

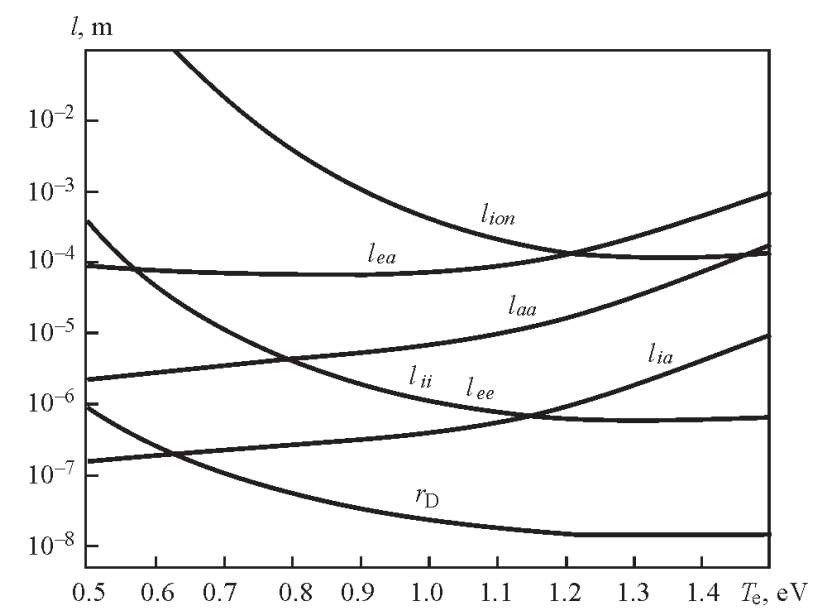

Figure 2. Typical lengths of free path of particles in atmospheric pressure argon plasma 
in time are neglected likewise. These speculations allow considering that axial component of electric field and axial gradient of plasma pressure are constant on channel section, and electric current on channel wall equals zero. A model of nonisothermal (two-temperature) ionization non-equilibrium plasma, including double ionization of atoms of the plasma gas (four-component plasma), is used in writing of the multi-fluid equations for the column and ionization region of arc plasma in the channel. The equitations of movement of plasma components in radial direction are written in a drift-diffusion approximation [5] and equality of axial velocities of heavy components is supposed in writing of corresponding equations in axial direction. Consideration of the processes taking place, the space charge layer and determination of characteristics of heat and electric interaction of arc plasma with channel wall is carried out by means of application of corresponding boundary conditions on this layer interface [3].

Mathematical model. Basic equations. For analysis of the physical processes, taking place in the studied plasma system, it is assumed that arc plasma is characterized by the following parameters: $n_{e}$ is the electrons concentration; $n_{n}, n_{i}, n_{i++}$ are the concentration of atoms of singly and doubly charged ions of the plasma gas, respectively; $v_{e}^{r}, v_{n}^{Z}$ are the radial and axial components of electrons velocity; $v_{n}^{r}, v_{n}^{Z} v_{i+}^{r}, v_{i+}^{Z}, v_{i++}^{r}, v_{i++}^{Z}$ are the radial and axial components of velocities of atoms and corresponding ions; $T_{\mathrm{e}}$ is the temperature of electrons; $T_{\mathrm{h}}$ is the temperature of heavy particles of plasma, being supposed similar for atoms and ions, but different from $T_{e}$. Then set of equations, describing stationary processes of mass, pulse and energy transfer in column plasma and ionization arc layer in asymptotic region of the cylindrical channel, can be written in the following way:

- Continuity equation for electrons, atoms, singly and doubly charged ions of arc plasma taking into account non-equilibrium ionization:

$$
\begin{gathered}
\frac{1}{r} \frac{\partial}{\partial r}\left(r n_{e} v_{e}^{r}\right)=k_{i 0} n_{n} n_{e}-k_{r 0} n_{e}^{2} n_{i+}+ \\
+k_{i 1} n_{i+} n_{e}-k_{r 1} n_{e}^{2} n_{i++} ; \\
\frac{1}{r} \frac{\partial}{\partial r}\left(r n_{n} v_{n}^{r}\right)=-k_{i 0} n_{n} n_{e}+k_{r 0} n_{e}^{2} n_{i+} ; \\
\frac{1}{r} \frac{\partial}{\partial r}\left(r n_{i+} v_{i+}^{r}\right)=k_{i 0} n_{n} n_{e}-k_{r 0} n_{e}^{2} n_{i+}- \\
\quad-k_{i 1} n_{i+} n_{e}+k_{r 1} n_{e}^{2} n_{i++} ; \\
\frac{1}{r} \frac{\partial}{\partial r}\left(r n_{i+} v_{i++}^{r}\right)=k_{i 1} n_{i+} n_{e}-k_{r 1} n_{e}^{2} n_{i++},
\end{gathered}
$$

where $k_{i \alpha}, k_{r \alpha}$ are the coefficients of ionization and recombination of atoms $(\alpha=0)$ and singly charged ions $(\alpha=1)$ [4]. Summing equations (2)-(4) and taking into account that particles of the plasma gas, assumed as inert one, do not accumulate on channel wall, the following is written:

$$
n_{i+} v_{i+}^{r}+n_{i++} v_{i++}^{r}+n_{n} v_{n}^{r}=0 .
$$

Multiplying equations (1), (3) and (4) by charge of corresponding particle and summing, by considering assumption on absence of electric current on channel wall, we receive

$$
j_{r}=e n_{i+} v_{i+}^{r}+2 e n_{i++} v_{i++}^{r}-e n_{e} v_{e}^{r}=0,
$$

where $j_{r}$ is the radial component of current density in arc plasma; $e$ is the electron charge. Thus, instead of equations (1)-(4) only two of them can be used by adding to them conditions (5) and (6).

Taking into account that plasma of column and ionization region of the arc is quasineutral, complete these equations by quasineutral condition, which in the case of four-component plasma is written as

$$
n_{e}=n_{i+}+2 n_{i++} .
$$

Besides, let's use condition of stability of total pressure of plasma along the channel section

$$
p=k\left[n_{e} T_{\mathrm{e}}+\left(n_{n}+n_{i+}+n_{i++}\right) T_{\mathrm{h}}\right],
$$

where $k$ is the Boltzmann constant;

- Equation of radial movement of electrons, atoms, singly and doubly charged ions, written in the drift-diffusion approximation (at convection members being neglected), but taking into account viscous members:

$$
\begin{gathered}
\frac{\partial\left(n_{e} k T_{\mathrm{e}}\right)}{\partial r}=\frac{2}{r} \frac{\partial}{\partial r}\left(r \eta_{e} \frac{\partial v_{e}^{r}}{\partial r}\right)- \\
-\frac{2}{3} \frac{\partial}{\partial r}\left(\frac{\eta_{e}}{r} \frac{\partial\left(r v_{e}^{r}\right)}{\partial r}\right)- \\
-\frac{2 \eta_{e} v_{e}^{r}}{r^{2}}+P_{e n}^{r}+P_{e i+}^{r}+P_{e i++}^{r}-e E_{r} n_{e} ; \\
\frac{\partial\left(n_{n} k T_{\mathrm{h}}\right)}{\partial r}=\frac{2}{r} \frac{\partial}{\partial r}\left(r \eta_{n} \frac{\partial v_{n}^{r}}{\partial r}\right)-\frac{2}{3} \frac{\partial}{\partial r}\left(\frac{\eta_{n}}{r} \frac{\partial\left(r v_{n}^{r}\right)}{\partial r}\right)- \\
-\frac{2 \eta_{n} v_{n}^{r}}{r^{2}}+P_{n i+}^{r}+P_{n e}^{r}+P_{n i++}^{r}+R_{n}^{r} ; \\
\frac{\partial\left(n_{i+} k T_{h}\right)}{\partial r}=\frac{2}{r} \frac{\partial}{\partial r}\left(r \eta_{i+} \frac{\partial v_{i+}^{r}}{\partial r}\right)- \\
-\frac{2}{3} \frac{\partial}{\partial r}\left(\frac{\eta_{i+}}{r} \frac{\partial\left(r v_{i+}^{r}\right)}{\partial r}\right)-\frac{2 \eta_{i+} v_{i+}^{r}}{r^{2}}+ \\
+P_{i+n}^{r}+P_{i+e}^{r}+P_{i+i++}^{r}+R_{i+}^{r}-R_{i++}^{r}+e E_{r} n_{i+} ; \\
\frac{\partial\left(n_{i++} k T_{\mathrm{h}}\right)}{\partial r}=\frac{2}{r} \frac{\partial}{\partial r}\left(r \eta_{i++} \frac{\partial v_{i++}^{r}}{\partial r}\right)- \\
-\frac{2}{3} \frac{\partial}{\partial r}\left(\frac{\eta_{i++}}{r} \frac{\partial\left(r v_{i++}^{r}\right)}{\partial r}\right)-\frac{2 \eta_{i++} v_{i++}^{r}}{r^{2}}+ \\
+P_{i++n}^{r}+P_{i++e}^{r}+P_{i++i+}^{r}+R_{i++}^{r}+2 e E_{r} n_{i++} ;
\end{gathered}
$$


-Equation of electron axial movement and resulting equation of axial movement of heavy particles:

$$
\begin{gathered}
\frac{1}{r} \frac{\partial}{\partial r}\left(r m_{e} n_{e} v_{e}^{z} v_{e}^{r}\right)=\frac{1}{r} \frac{\partial}{\partial r}\left(r \eta_{e} \frac{\partial v_{e}^{z}}{\partial r}\right)+ \\
+P_{e n}^{z}+P_{e i+}^{z}+P_{e i++}^{z}-e E_{z} n_{e} ; \\
\frac{\partial \sum_{\alpha} n_{\alpha} k T_{\mathrm{h}}}{\partial z}+\frac{1}{r} \frac{\partial}{\partial r}\left(r \sum_{\alpha} m_{\alpha} n_{\alpha} v_{h}^{z} v_{\alpha}^{r}\right)= \\
=\frac{1}{r} \frac{\partial}{\partial r}\left(r \sum_{\alpha} \eta_{\alpha} \frac{\partial v_{h}^{z}}{\partial r}\right)+P_{n e}^{z}+P_{i+e}^{z}+P_{i++e}^{z} \\
+e E_{z}\left(n_{i+}+2 n_{i++}\right), \quad \alpha=n, i+, i++.
\end{gathered}
$$

The following designations are used in equations (9)-(14): $\eta_{\alpha}, m_{\alpha}$ are the coefficients of dynamic viscosity and mass of particles of plasma component $\left(\alpha=e, n, i^{+}, i^{++}\right) ; R_{\alpha \beta}^{r}, R_{\alpha \beta}^{z}$ is the radial and axial components of exchange terms $\left(\alpha \neq \beta=e, n, i^{+}, i^{++}\right)$ corresponding to elastic collision of particles [1]; $R_{\alpha}^{r}$ are the radial components of exchange terms $(\alpha=n$, $i+, i++)$, corresponding to inelastic collisions [1]; $E_{r}$, $E_{z}$ are the radial and axial components of electric field in arc plasma;

- Movement equation shall be completed by conditions of arc total current conservation

$$
I=2 \pi \int_{0}^{R}\left|j_{z}\right| r d r,
$$

where $j_{z}=e n_{i+} v_{i+}^{z}+2 e n_{i++} v_{i++}^{z}-e n_{e} v_{e}^{z}$ is the axial component of density of electric current in plasma as well as condition of conservation of mass consumption of plasma gas through channel cross section

$$
G_{M}=\rho_{0} G=2 \pi \int_{0}^{R} r \sum_{\alpha=n, i+, i++} m_{\alpha} n_{\alpha} v_{\alpha}^{z} d r,
$$

where $\rho_{0}$ is the density of plasma gas under normal conditions;

- Equation of electron energy transfer and total equation of energy of heavy particles are written in the following way:

$$
\begin{gathered}
\frac{1}{r} \frac{\partial}{\partial r}\left(v_{e}^{r} \frac{5}{2} n_{e} k T_{\mathrm{e}}\right)=\frac{1}{r} \frac{\partial}{\partial r}\left(r \lambda_{e} \frac{\partial T_{\mathrm{e}}}{\partial r}\right)+ \\
+\sum_{\alpha}\left(Q_{e \alpha}+G_{e \alpha}\right)-e\left(E_{z} v_{e}^{z}+E_{r} v_{e}^{r}\right) n_{e}-Q_{r}, \\
\frac{1}{r} \frac{\partial}{\partial r} \sum_{\alpha}\left(v_{\alpha}^{r}\left(\frac{5}{2} n_{\alpha} k T_{\mathrm{h}}+\frac{1}{2} m_{\alpha} n_{\alpha} v_{\alpha}^{2}\right)\right)= \\
=\frac{1}{r} \frac{\partial}{\partial r}\left(r \sum_{\alpha} \lambda_{\alpha} \frac{\partial T_{\mathrm{h}}}{\partial r}\right)+\sum_{\alpha} Q_{\alpha e}+ \\
+e\left(E_{z} v_{i+}^{z}+E_{r} v_{i+}^{r}\right) n_{i+}+2 e\left(E_{z} v_{i++}^{z}+E_{r} v_{i++}^{r}\right) n_{i++}, \\
\alpha=n . i+. i++.
\end{gathered}
$$

where $\lambda_{\alpha}$ are the coefficients of heat conduction of plasma component $\left(\alpha=e, n, i^{+}, i^{++}\right) ; Q_{\alpha \beta}$ are the exchange terms $(\alpha \neq \beta=e, n, i+, i++)$ corresponding to particle elastic collisions [1]; $Q_{e \alpha}$ are the exchange terms $(\alpha=i+, i++)$ corresponding to non-elastic collisions of electrons with heavy particles [1]; $Q_{r}$ is the electron energy loss for heat irradiation.

Work [9] gives the formulae for calculation of coefficients of ionization and recombination, collision frequency, transportation coefficients and radiation losses of electron energy, being included in equations (1)-(4), (9)-(14), (17) and (18) for further studied atmospheric (high-pressure) plasma. Explicit form of the exchange elements, included in (9)-(14), (17) and (18), are also given there.

Boundary conditions. Taking into account used approximation on axial symmetry of studied plasma system, the following can be assumed on channel axis:

$$
\begin{gathered}
\left.\frac{\partial n_{\alpha}}{\partial r}\right|_{r=0}=0, \quad \alpha=i+, i++; \\
\left.v_{\alpha}^{r}\right|_{r=0}=0, \quad \alpha=i+, i++; \\
\left.\frac{\partial v_{\alpha}^{z}}{\partial r}\right|_{r=0}=0, \quad \alpha=n, i+, i++; \\
\left.\frac{\partial T_{\mathrm{e}}}{\partial r}\right|_{r=0}=\left.\frac{\partial T_{\mathrm{h}}}{\partial r}\right|_{r=0}=0 .
\end{gathered}
$$

In order to set boundary conditions on channel wall, to be more accurate on interface of ionization region under the layer of space charge, the following is done. Using equations (5), (6), (9)-(11), the flow of singly charged ions on indicated interface can be written in form of

$$
\begin{gathered}
\left.n_{i+} v_{i+1}^{r}\right|_{r=r_{s}}=\left.D_{i+i+}^{T} \frac{\partial\left(T_{\mathrm{e}}+T_{\mathrm{h}}\right)}{\partial r}\right|_{r=r_{s}}+ \\
+\left.D_{i+i+} \frac{\partial n_{i+}}{\partial r}\right|_{r=r_{s}}+\left.D_{i+i++}^{T} \frac{\partial\left(2 T_{\mathrm{e}}+T_{\mathrm{h}}\right)}{\partial r}\right|_{r=r_{s}}+\left.D_{i+i++} \frac{\partial n_{i++}}{\partial r}\right|_{r=r_{s}},
\end{gathered}
$$

where $D_{\mathrm{a} \beta}, D_{\alpha \beta}^{T}, \alpha=i+, \beta=i+, i++$ are the transportation coefficients, explicit form of which is given in appendix. At that, velocity of singly charged ions on interface of space charge layer can be taken equal to Bohm velocity, which has the following form in the case of three-component plasma [10]:

$$
\left.v_{i+}^{r}\right|_{r=r_{s}}=\left.\sqrt{\frac{k\left(T_{\mathrm{e}}+T_{\mathrm{h}}\right)}{m_{i+}}}\right|_{r=r_{s}} .
$$

Assuming that gradient of concentration of doubly charged ions on indicated interface equals zero:

$$
\left.\frac{\partial n_{i++}}{\partial r}\right|_{r=r_{s}}=0
$$

their flow can be determined, assuming, for example, the velocity of doubly charged ions on external interface of pre-layer being equal

$$
\left.v_{i++}^{r}\right|_{r=r_{s}}=\left.\sqrt{\frac{k\left(2 T_{\mathrm{e}}+T_{\mathrm{h}}\right)}{m_{i++}}}\right|_{r=r_{\mathrm{s}}},
$$


that together with relationship (24) corresponds to Bohm criterion in the case of four-component plasma.

Let's write the following for axial components of velocities of plasma heavy particles close to channel wall, taking into account «adhesion» condition:

$$
\left.v_{\alpha}^{z}\right|_{r=r_{s}}=0, \alpha=n, i+, i++.
$$

Boundary condition for electron temperature is set in from of [3]

where

$$
\begin{gathered}
\left(v_{e}^{r} \frac{5}{2} n_{e} k T_{\mathrm{e}}+\lambda_{e} \frac{\partial T_{\mathrm{e}}}{\partial r}\right)_{r=r_{s}}= \\
=\left.j_{e}^{r}\left(2 k T_{\mathrm{e}}+e \Delta \phi_{\mathrm{s}}\right)\right|_{r=r_{\mathrm{s}}},
\end{gathered}
$$

$$
\Delta \phi_{s}=-\left.\frac{k T_{\mathrm{e}}}{2 e} \ln \frac{2 \pi m_{e}\left(T_{\mathrm{e}}+T_{\mathrm{h}}\right)}{m_{i+} T_{\mathrm{e}}}\right|_{r=r_{s}}
$$

of potential drop in space charge layer [3]. Temperature of plasma heavy particles close to wall can be set with sufficient accuracy equal to the temperature of wall channel:

$$
\left.T_{\mathrm{h}}\right|_{r=r_{\mathrm{s}}}=T_{\mathrm{w}} \text {. }
$$

The important characteristic of the processes of heat and electric interaction of arc plasma with plasmatron channel wall are heat flow from plasma on channel wall (see, for example [3]):

$$
q_{\mathrm{w}}=\left.\left\{\begin{array}{l}
j_{i+}^{r}\left[k\left(2 T_{\mathrm{h}}+\frac{1}{2} T_{\mathrm{e}}\right)+e \Delta \phi_{\mathrm{s}}+E_{1}\right]+ \\
+j_{i++}^{r}\left[k\left(2 T_{\mathrm{h}}+T_{\mathrm{e}}\right)+2 e \Delta \phi_{\mathrm{s}}+E_{2}\right]+ \\
+2 j_{e}^{r} k\left(T_{\mathrm{e}}-T_{\mathrm{w}}\right)
\end{array}\right\}\right|_{r=r_{s}}
$$

as well as potential drop between arc plasma and wall, which consists of voltage drop in column and ionization region of arc plasma as well as potential drop in space charge layer, determined by expression (29).

Described above mathematical model of the fourcomponent plasma of electric arc in asymptotic region of plasmatron channel wall can be easily reduced for arc modelling in the channel under those modes of its arcing when formation of the doubly charged ions in arc plasma can be neglected (three-component plasma). For this, in the model equations, corresponding to boundary conditions, and expressions for determination of transportation coefficients of plasma component as well as radiation loss of electron energy, it is enough to set $n_{i++}=0$, coefficient of ionization of singly charged ions in continuity equations equate to zero and drop the exchange terms, corresponding to collisions at participation or appearance of the doubly charged ions, in the equations of movement and energy transfer.
Procedure and algorithm of problem solving. Stationary solution (relaxation) method is used in numerical solution of the stated problem. For this reason the indicial equations (1)-(4), (9)-(14), (17), (18) are completed with corresponding non-stationary members and written in dimensionless form. The following values are taken as scales of temperature, concentration, length, velocity and time:

$$
\begin{gathered}
\bar{T}=1 \mathrm{eV} ; \bar{n}=10^{23} \mathrm{~m}^{-3} ; \bar{L}=10^{-4} \mathrm{~m} ; \\
\bar{v}=10^{3} \mathrm{~m} / \mathrm{s} ; \bar{t}=10^{-7} \mathrm{~s} .
\end{gathered}
$$

Obtained equations are solved numerically by finite element method. At that an implicit scheme with secondary approximation on nonlinear finitevolumetric mesh [11], taking into account linear approximation of the boundary conditions (19-28) and (30) written in dimensionless form, is used. Uniform of channel section distributions of temperature, velocities and concentrations of plasma component as well as electric field, corresponding to equations (1)(18) at neglect of radial nonuniformity of the system, are used as initial conditions.

Computer realization of the described algorithm is carried out in two steps, namely: 1 - pre-processor, realized in software package Matlab, provides for set of system initial parameters and determination of corresponding to them characteristics of arc plasma in the nozzle channel being assumed as radially uniform, and 2 - post-processor, realized in software environment Fortran, provides for numerical solution of a set of algebraic equations approximating differential equations (3), (4), (9), (10)-(14), (17), and (18). Method of paralleling for four cores by means of program setting of corresponding part of calculation region to each core (processor) is used in computations. It significantly increases speed of numerical results obtaining.

Modelling results. The following system parameters were used for calculation of characteristics of arc plasma in the plasmatron nozzle channel: arc current $I=50,100,200 \mathrm{~A}$; channel radius $R=$ $=1.2 \mathrm{~mm}$; volumetric consumption of plasma gas (Ar) $G=2.51 / \mathrm{min}$; temperature of channel wall $T_{w}=$ $=1160 \mathrm{~K}$. Figures $3-8$ show the results of calculation of radial distributions of characteristics in studied plasma.

Firstly, let's study effect of arc current and channel radius on distributions of electron temperature and temperature of plasma heavy components without notice of doubly charged ions (three-component plasma) in it. As follows from calculation results in Figure 3, the temperatures of electrons and plasma heavy particles in arc column are virtually matched, i.e. arc plasma in central region of the channel is 

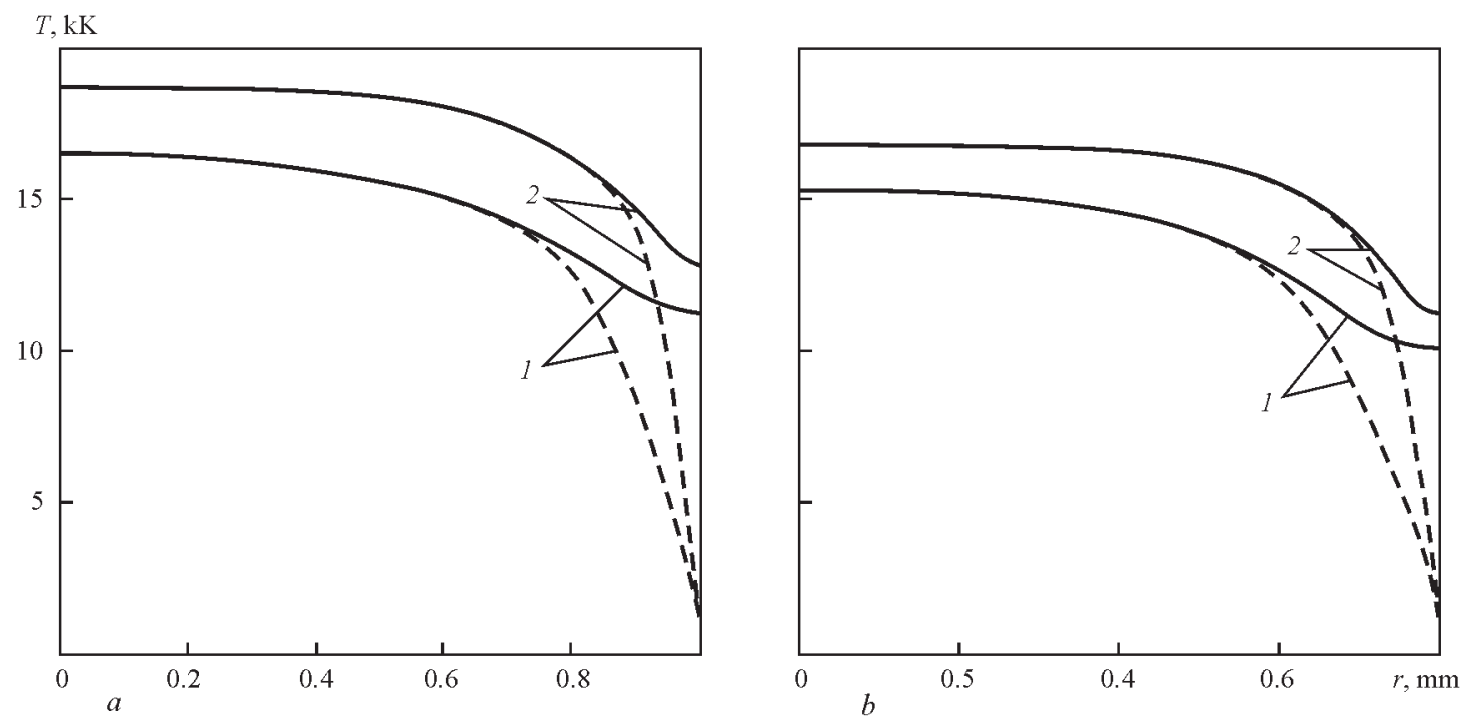

Figure 3. Radial distributions of temperature of electrons (solid curves) and heavy particles (dashed) of arc plasma in plasmatron nozzle channel: $a-R=1 \mathrm{~mm}, G=2 \mathrm{l} / \mathrm{min} ; 1-I=50 \mathrm{~A} ; 2-100 \mathrm{~A} ; b-R=2 \mathrm{~mm}, G=5 \mathrm{l} / \mathrm{min} ; 1-I=100 \mathrm{~A} ; 2-200 \mathrm{~A}$

thermally equilibrium, besides its temperature rises at increase of arc current and decrease of channel radius (see also $[7,8]$ ). $T_{\mathrm{e}}$ significantly exceeds $T_{\mathrm{h}}$ in the vicinity to channel wall, at that size of region of thermally non-equilibrium plasma, determining prelayer thickness, is vice versa reduced at rise of $I$ and decrease of $R$ (see Figure 3). The reason for higher in comparison with $T_{\mathrm{h}}$ values of temperature of electrons in the near-wall region of arc is a reduction of efficiency of energy exchange between electron component and heavy particles, resulting in temperature equalization.

Figure 4 shows calculation distributions of level of ionization non-equilibrium of arc plasma on the channel wall, determined as $\delta=n_{e} / n_{e}^{\text {Saha }}$ (where $n_{e}^{\text {Saha }}$ is the equilibrium concentration of electrons, calculated based on Saha equation with electron temperature, quasilinearity condition and partial pressure law). Calculation data indicate that

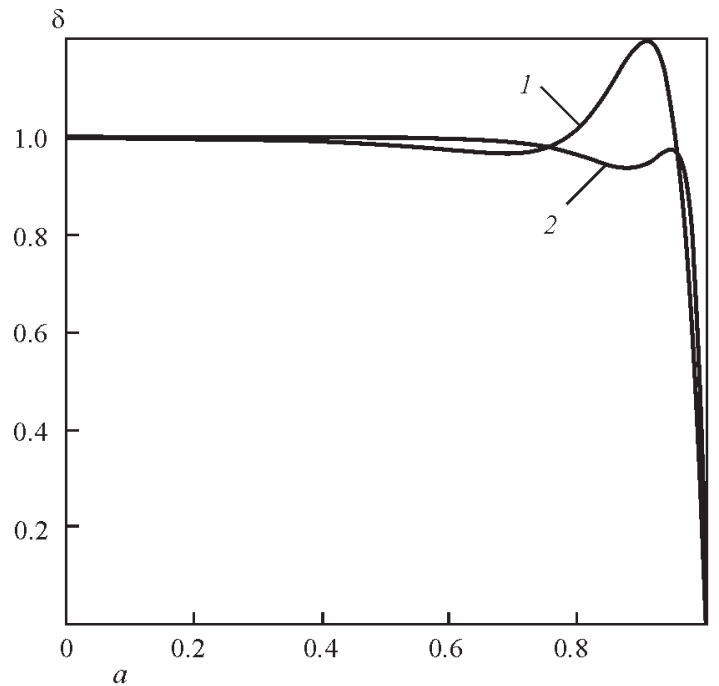

arc column plasma with high level of accuracy has not only thermal (see Figure 3), but also ionization equilibrium. Arc plasma in the pre-layer is significantly non-equilibrium, moreover $\delta$ value, characterizing level of non-equilibrium ionization, firstly somewhat increases and then rapidly drops as far as approaching to channel wall (concentration of electrons on interface of space charge is two-three order lower than equilibrium one). An important circumstance in this case is the fact that the regions of ionization and thermal plasma non-equilibrium are virtually matched under studied parameters of the system, and their sizes reduce with increase of $I$ and decease of $R$ (see Figures 3 and 4). It should be noted here that the size of region of thermal and ionization non-equilibrium of arc plasma in the plasmatron nozzle channel makes $20-35 \%$ of $R$ value.

Doubly charged ions, presence of which can be considered in scope of the four-component plasma

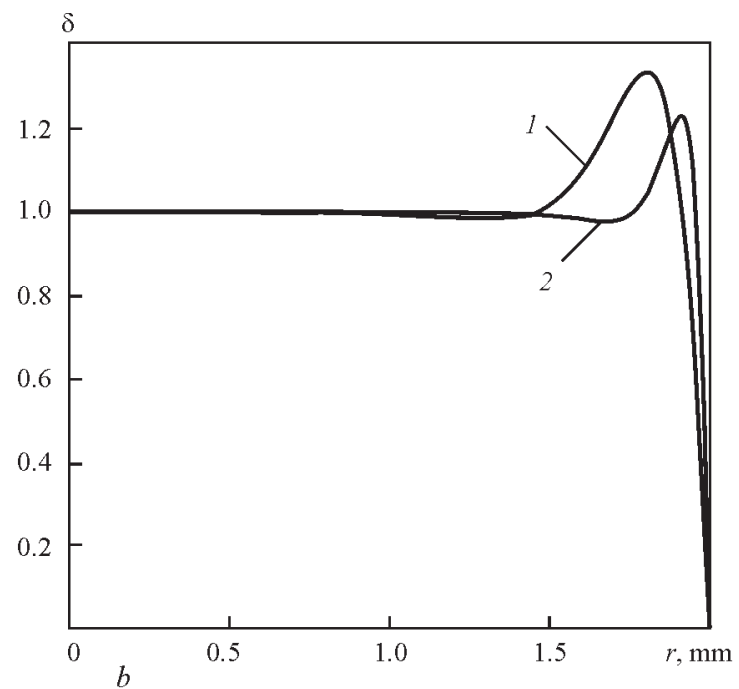

Figure 4. Radial distributions of level of ionization non-equilibrium of arc plasma in plasmatron nozzle channel: $a-R=1 \mathrm{~mm}, G=$ $=2 \mathrm{l} / \mathrm{min} ; 1-I=50 \mathrm{~A} ; 2-100 \mathrm{~A} ; b-R=2 \mathrm{~mm}, G=5 \mathrm{l} / \mathrm{min} ; 1-I=100 \mathrm{~A} ; 2-200 \mathrm{~A}$ 

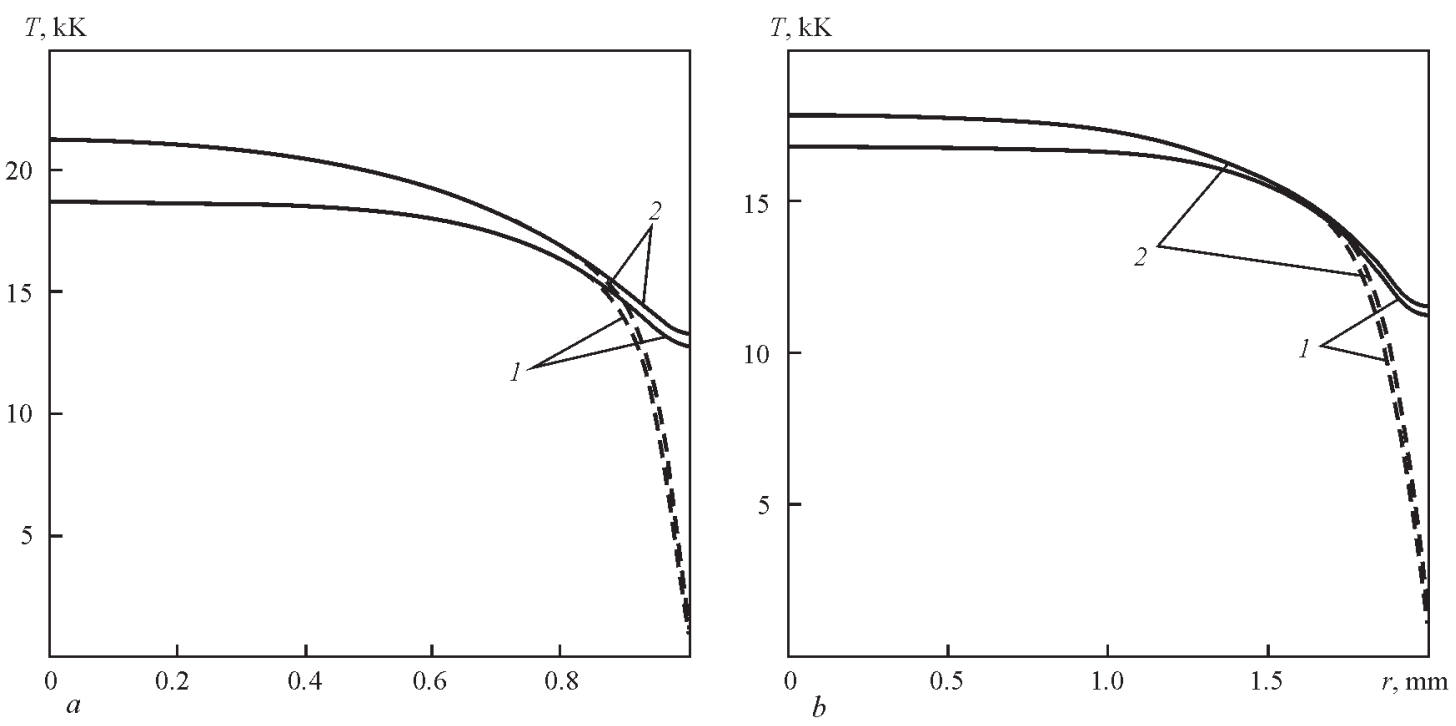

Figure 5. Radial distributions of temperature of electrons (solid curves) and heavy particles (dashed) of arc plasma in plasmatron nozzle channel: $a-R=1 \mathrm{~mm}, G=2$ 1/min, $I=100 \mathrm{~A} ; b-R=2 \mathrm{~mm}, G=51 / \mathrm{min}, I=200 \mathrm{~A} ; 1$ - three-; 2 - four-component plasma

model, make significant contribution in formation of arc plasma characteristics with rise of their temperature in the nozzle channel at $I$ increase. Figure 5 shows comparison of calculation distribution of temperature of electrons and arc plasma heavy particles on nozzle section, obtained using the models of three- and four-component plasma. As it follows from presented results, the temperatures of plasma component in the arc column, calculated taking into account doubly charged ions, significantly exceed corresponding values of three-component plasma. At that, arc column plasma is virtually equilibrium. Indicated difference reduces in the near-wall region, up to virtually complete matching of temperatures (separately for electrons and for heavy plasma component). The reason for this is reduction of role

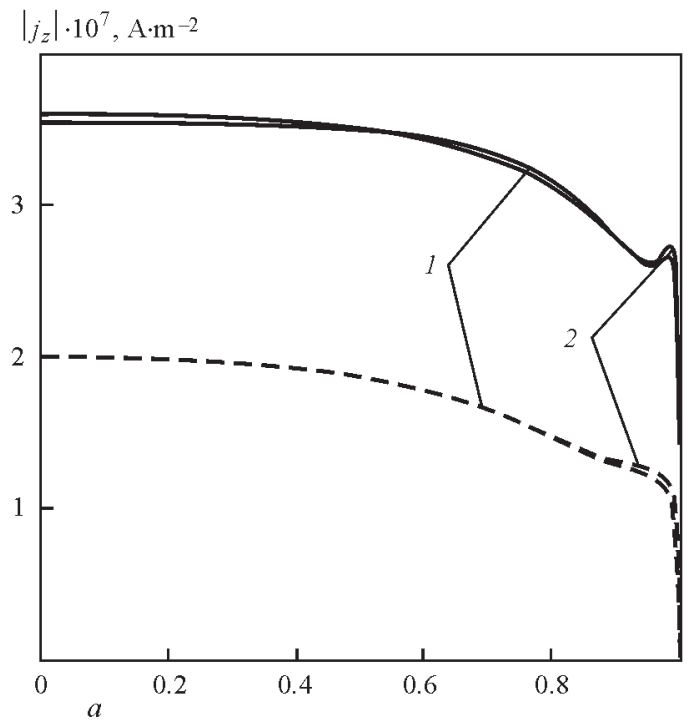

of doubly charged ions in the vicinity to channel wall, concentration of which here is negligibly small.

Figure 6 represents the calculation dependencies of radial distributions of absolute values of axial component of electric current density for arc in channel. Local increase of $\left|j_{z}\right|$ in the vicinity to channel wall can be noted together with obvious increase of current density at $I$ rise and $R$ decrease (see also $[7,8]$ ). It becomes very vividly apparent at large currents and small radiuses of the channel (see solid curves in Figure 6, $a$ ) and is caused by increase of axial component of electron velocity in the plasma pre-layer due to reduction of frequency of their collision with heavy particles. Difference of calculation results of electric current density in scope of models for three- and four-component plasma seems to be virtually unessential.

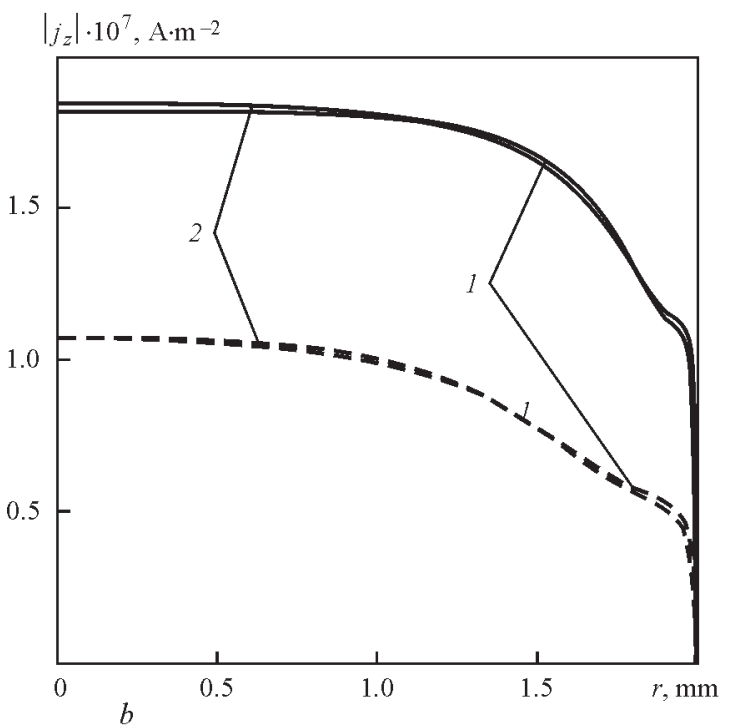

Figure 6. Radial distributions of density of electric current for arc in plasmatron nozzle channel: $a-R=1 \mathrm{~mm}, G=21 / \mathrm{min}, I-50$ (dashed curves) and $100 \mathrm{~A}$ (solid); $b-R=2 \mathrm{~mm}, G=5 \mathrm{l} / \mathrm{min}, I=100$ (dashed) and $200 \mathrm{~A}$ (solid); 1 - three-; 2 - four-component plasma 

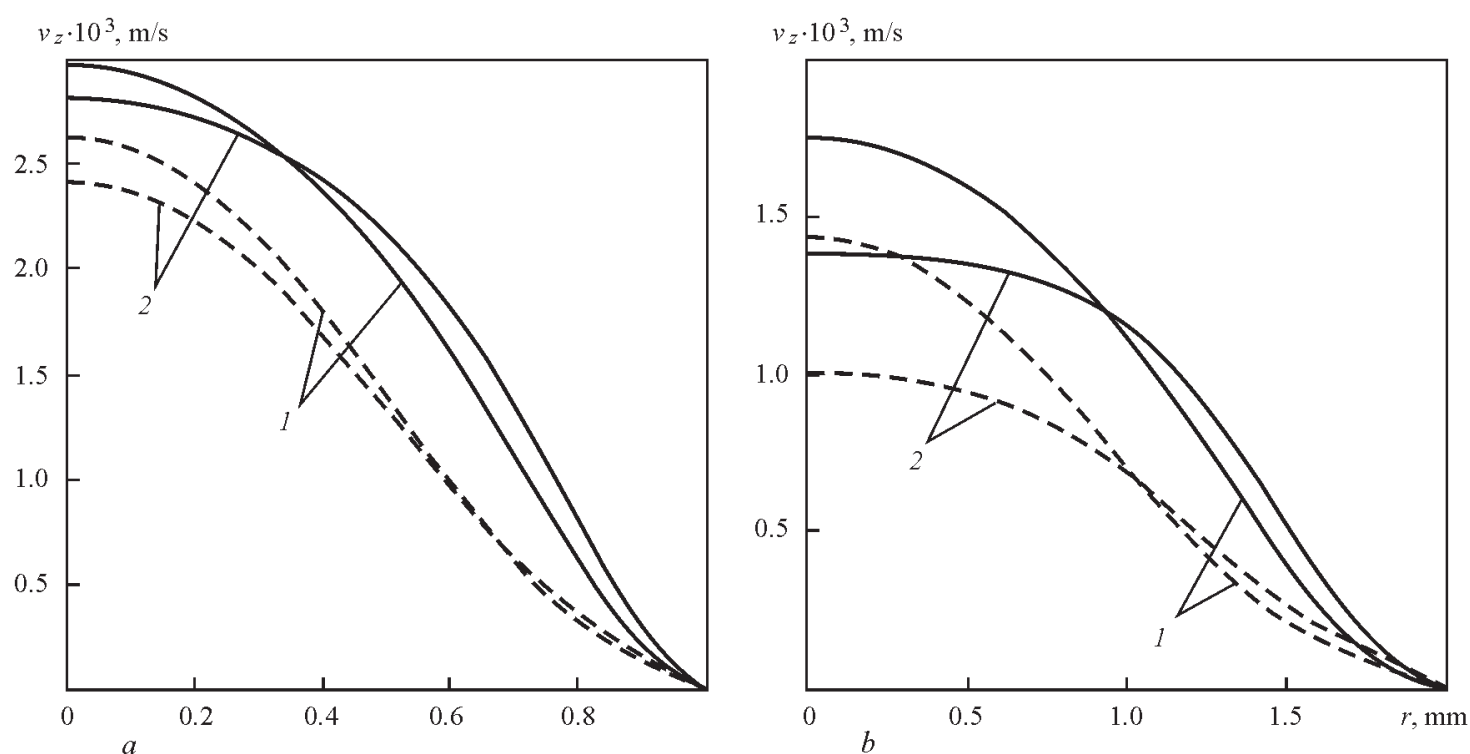

Figure 7. Radial distributions of mass-average velocity of arc plasma in plasmatron nozzle channel: $a-R=1 \mathrm{~mm}, G=21 / \mathrm{min}, I-50$ (dashed) and $100 \mathrm{~A}$ (solid); $b-R=2 \mathrm{~mm}, G=5 \mathrm{l} / \mathrm{min}, I=100$ (dashed) and $200 \mathrm{~A}$ (solid); 1 — three-; 2 — four-component plasma
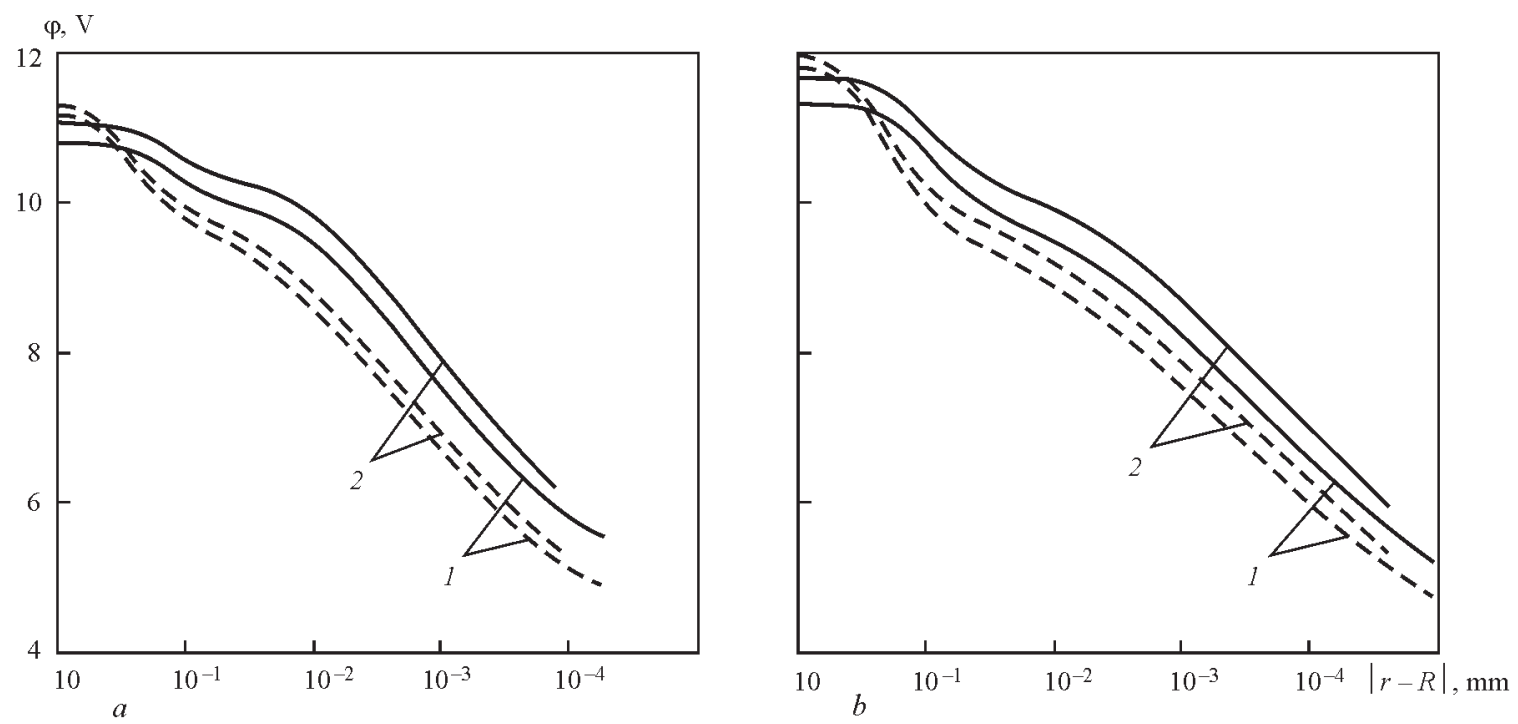

Figure 8. Radial distributions of electric potential of arc plasma in plasmatron nozzle channel: $a-R=1 \mathrm{~mm}, G=21 / \mathrm{min}, I-50$ (dashed) and $100 \mathrm{~A}$ (solid); $b-R=2 \mathrm{~mm}, G=5 \mathrm{l} / \mathrm{min}, I=100$ (dashed) and $200 \mathrm{~A}$ (solid); 1 — three-; 2 - four-component plasma

Let's study the effect of arc current, channel radius and consumption of plasma gas on radial distribution of axial component of mass-average velocity

$$
v_{z}=\sum_{\alpha=n, i+, i++} m_{\alpha} n_{\alpha} v_{\alpha}^{z} / \sum_{\alpha=n, i+, i++} m_{\alpha} n_{\alpha}
$$

of arc plasma in the channel. Plasma velocity on channel axis has virtually proportional rise at increase of relationship of plasma gas consumption to channel cross-section area (see also [7]) and to less degree at arc current rise. Difference between values of $v_{z}$, calculated in scope of models for three- and fourcomponent plasma, in contrast to $\left|j_{z}\right|$, appears to be significant (Figure 7) and mainly developed in the arc column. At that, $v_{z}$ value for the three-component plasma in near-axis region of the arc column exceeds corresponding values in the case of four-component plasma and it is, vice versa, in near-wall region. This effect is related with higher values of viscosity factor

Heat flow from plasma on wall channel and potential drop in space charge layer

\begin{tabular}{|c|c|c|c|c|c|c|c|}
\hline \multirow{2}{*}{ Parameter } & \multirow{2}{*}{$\begin{array}{c}\text { Calculation } \\
\text { data }\end{array}$} & \multicolumn{2}{|c|}{$I=50 \mathrm{~A}$} & \multicolumn{2}{c|}{$I=100 \mathrm{~A}$} & \multicolumn{2}{c|}{$I=200 \mathrm{~A}$} \\
\cline { 2 - 8 } & 3-comp. & 4-comp. & 3-comp. & 4-comp. & 3-comp. & 4-comp. \\
\hline $\begin{array}{l}R=1 \mathrm{~mm}, \\
G=2 \mathrm{l} / \mathrm{min}\end{array}$ & $q_{\mathrm{w}}, \mathrm{MW} / \mathrm{m}^{2}$ & 2.115 & 2.184 & 11.730 & 13.140 & - & - \\
\cline { 2 - 8 } & $\Delta \varphi_{s}, \mathrm{~V}$ & 4.46 & 4.57 & 5.10 & 5.31 & - & - \\
\hline $\begin{array}{l}R=2 \mathrm{~mm}, \\
G=5 \mathrm{l} / \mathrm{min}\end{array}$ & $q_{\mathrm{w}}, \mathrm{MW} / \mathrm{m}^{2}$ & - & - & 0.260 & 0.221 & 1.614 & 1.448 \\
\cline { 2 - 8 } & $\Delta \varphi_{s}, \mathrm{~V}$ & - & - & 4 & 4.08 & 4.45 & 4.60 \\
\hline
\end{tabular}




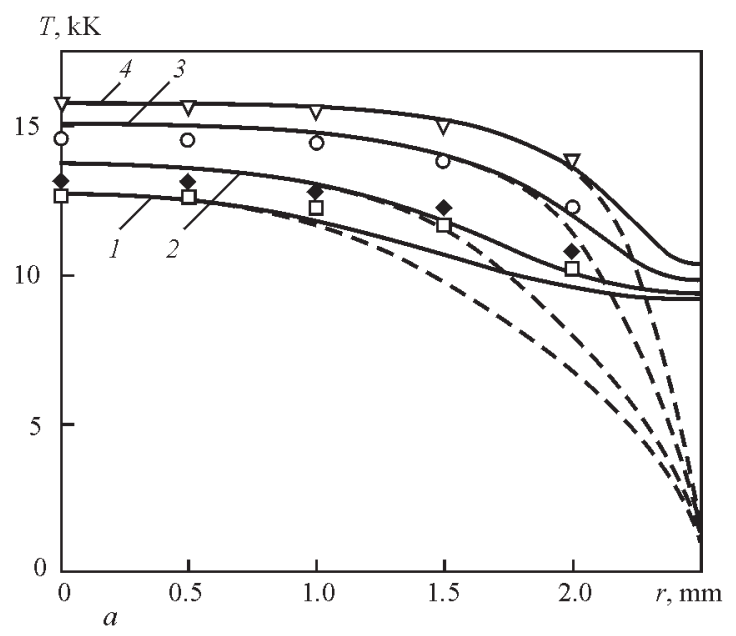

$n_{e} \cdot 10^{23}, \mathrm{~m}^{-3}$

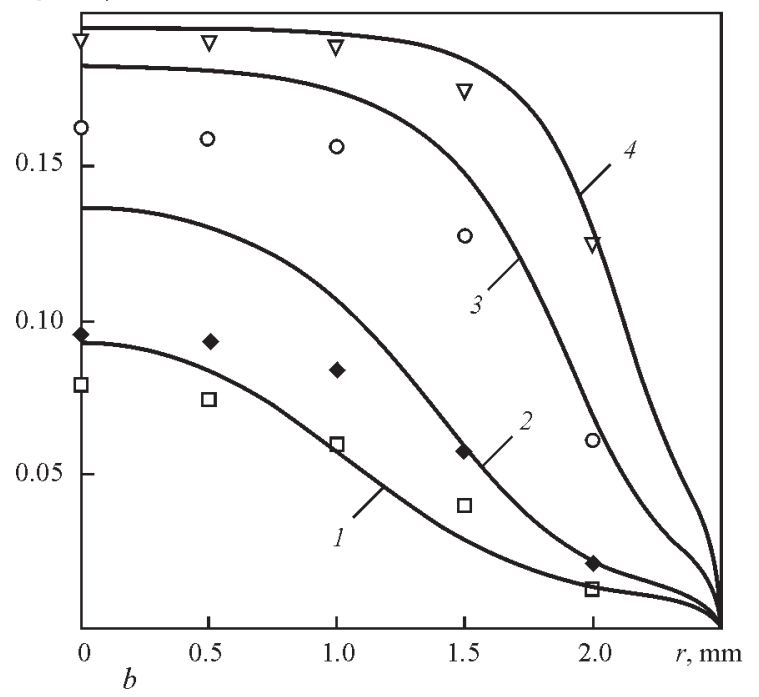

$E_{z}, \mathrm{kV} / \mathrm{m}$

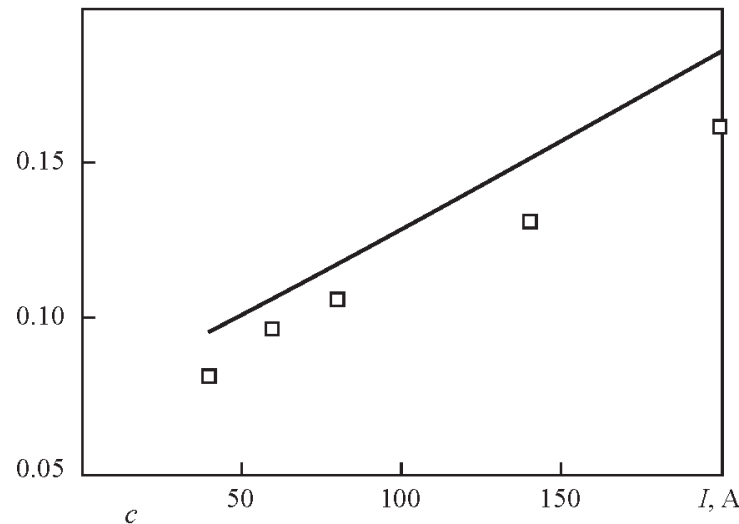

Figure 9. Comparison of calculation data (solid curves) with experimental (markers) for arc in $2.5 \mathrm{~mm}$ radius channel, blown out by argon flow with $0.07 \mathrm{l} / \mathrm{min}$ consumption at $60(-), 80(+)$, $140(\mathrm{o})$ and $200 \mathrm{~A}(\Delta)$ currents: $a$ - radial distributions of electron temperature; $b$ - radial distributions of electron concentration; $c$ - dependence of axial component of electric field on arc current for the four-component plasma in comparison with three-component plasma.

Now let's come to consideration of the distribution of electric potential of arc plasma on channel section (Figure 8). As it follows from calculation data presented, plasma potential on channel axis exceeds wall potential, conventionally taken as zero, by $11-$ $12 \mathrm{~V}$. Moreover, in the case of application of fourcomponent plasma the calculation values of potential on arc column axis appear to be somewhat higher than in use of three-component model. It should be noted that specified values of potential difference between arc axis and channel wall are the sum of voltage drop in the arc column and plasma pre-layer with voltage drop in space charge layer calculated on formula (29), corresponding values of which are given in the Table. It also provides for the values of such important from practical point of view characteristic as heat flow from plasma to channel wall, calculated on formula (31).

Model verification. Adequacy of the developed model of processes of energy, pulse, mass and charge transfer in non-equilibrium plasma of electric arc in the plasmatron nozzle channel is considered by means of comparison of the calculation results, produced in scope of three-component plasma model, with available experimental data [8]. The calculation of characteristics of arc plasma was carried out under system parameters corresponding to experiment conditions [8], namely at arc current $I=40-200$ A; channel radius $R=2.5 \mathrm{~mm}$; volumetric consumption of plasma gas (Ar) $G=0.071 / \mathrm{min}$; temperature of channel wall $T_{\mathrm{w}}=500 \mathrm{~K}$.

Figure 9 represents the results of calculation of radial distribution of temperature of electrons and heavy particles, concentration of plasma electrons as well as dependence of intensity of longitudinal electric field on arc current. Comparison of the calculation values of temperature and concentration of electrons with given in this Figure experimental data shows their totally satisfactory correspondence (maximum relative errors make 5 and $35 \%$, respectively). The maximum relative error in comparison of the calculation values of electric field axial component with experimental ones does not exceed $10 \%$. It indicates sufficiently high accuracy of results, obtained with the help of proposed model.

\section{Conclusions}

The mathematical model was proposed for nonequilibrium plasma of electric arc in asymptotic region of the channel, blown out by laminar flow of inert gas. It is used as a basis for numerical analysis of characteristics of arc plasma in the nozzle channel of direct plasmatron at different values of arc current, channel radius and consumption of plasma gas, that allows making the following conclusions:

1. Results of calculation of characteristics of studied plasma in scope of drift-diffusion approximation are virtually matched with the modelling results based 
on complete set of multi-fluid equations (taking into account convective terms), that allows recommending such a simplified approach for modelling of processes of energy, pulse, mass and charge transfer in nonequilibrium arc plasma.

2. Calculations of characteristics of argon arc plasma in the plasmatron nozzle channel at arc current and channel radius in $I / R>50 \mathrm{~A} / \mathrm{mm}$ range require taking into account plasma doubly charged ions. Such a consideration allows also calculating characteristics of plasma arc when it is effected by focused beam of $\mathrm{CO}_{2}$-laser irradiation, propagating along the channel axis, under conditions of laser-plasma welding.

3. The calculations showed that in contrast to channel central regions, where arc plasma is virtually equilibrium, plasma in the near-wall region appears to be non-equilibrium, moreover thermally as well as ionization. Besides, potential of channel wall is significantly lower than existing value of arc plasma potential on channel axis. Corresponding difference of the potentials can make 11-12 $\mathrm{V}$ depending on selected values of arc current, channel radius and plasma gas consumption.

4. Comparison of results of modelling of characteristics in non-equilibrium plasma of argon arc in the plasmatron nozzle channel with available experimental data showed their totally satisfactory correspondence, that indicate adequacy of the proposed mathematical model.

This work was carried out under financial support in scope of program for foreign experts in PRC No.WQ20124400119, project of research innovation group of Guangdong province No.201101C0104901263 and international project of Ministry of Science and Technology of PRC No.2013DFR70160.

1. Meier, E.T., Shumlak, U. (2012) A general nonlinear fluid model for reacting plasma-neutral mixtures. Physics of Plasma, 19, 072508.

2. Benilov, M.S. (1995) The ion flux from a thermal plasma to a surface. J. Phys. D: Appl. Phys., 28, 286-294.

3. Benilov, M.S., Marotta, A. (1995) A model of the cathode region of atmospheric pressure arcs. Ibid., 28, 1869-1882.

4. Almeida, N.A., Benilov, M.S., Naidis, G.V. (2000) Simulation of the layer of non-equilibrium ionization in a high-pressure argon plasma with multiply-charged ions. Ibid., 33, 960-967.

5. Almeida, N.A., Benilov, M.S., Naidis, G.V. (2008) Unified modelling of near-cathode plasma layers in high-pressure arc discharges. Ibid., 41, 245201.

6. Almeida, N.A., Benilov, M.S., Hechtfischer, U. (2009) Investigating near-anode plasma layers of very high-pressure arc discharges. Ibid., 42, 045210.

7. Engelsht, V.S., Gurovich, V.Ts., Desyatkov, G.A. et al. (1990) Low-temperature plasma. Vol. 1: Theory of electric arc column. Novosibirsk: Nauka.

8. Beulens, J.J., Milojevic, D., Schram, D.C. et al. (1991) A twodimensional nonequilibrium model of cascaded arc plasma flows. Phys. Fluids B, 3(9), 2548-2557.

9. Ignatov, A.V., Semenov, I.L. (2015) Characteristics of nonequilibrium arc plasma in channel of plasmatron nozzle. In: Proc. of $8^{\text {th }}$ Int. Conf. of Junior Scientists on Welding and Related Technologies (Vorzel, Ukraine, 2015).

10. Benilov, M.S. (1996) Multifluid equations of a plasma with various species of positive ions and the Bohm criterion. $J$. Phys. D: Appl. Phys., 29, 364-368.

11. Manteuffel, T.A., White, A.B. (1986) The numerical solution of second-order boundary value problems on nonuniform meshes. Mathematics of Computation, 47, 511-535.

Received 24.09.2015 